\title{
Arava Treatment, between Beneficial Action on Joint Inflammation and Side Effects on Liver, Myocardium and Kidney in Experimental Murine Arthritis
}

\author{
Mihaela Manole1, Codruta Badescu², Manuela Ciocoiu1, Magda Badescu ${ }^{1 *}$, Mihaiela Chicu ${ }^{1}$ \\ ${ }^{1}$ Pathophysiology Department, University of Medicine and Pharmacy “Grigore T. Popa”, Iasi, Romania \\ ${ }^{2}$ Internal Medicine Clinic, St. Spiridon Hospital, Iasi, Romania \\ Email: ${ }^{*}$ magda.badescu@gmail.com
}

Received 20 May 2014; revised 3 July 2014; accepted 15 July 2014

Copyright (C) 2014 by authors and Scientific Research Publishing Inc.

This work is licensed under the Creative Commons Attribution International License (CC BY).

http://creativecommons.org/licenses/by/4.0/

(c) (i) Open Access

\begin{abstract}
The purpose of the experiment was the follow-up in time of the course of joint inflammation phenomena in laboratory animals (white female adult Wistar rats) by determining particular biological, hematological, radiological, osteodensitometric, immunological and anatomic-pathological parameters and by assessing the effects of Leflunomide (Arava) on the course of the disease. ARAVA $^{\circledR}$ (Leflunomide) is a pyrimidine synthesis inhibitor. The experimental study was conducted for 8 weeks. We have also assessed the side effects of the therapy on their liver, myocardium and kidney. Leflunomide therapy improved the course of the clinical and paraclinical parameters, but it did not cure the condition. The positive results in the joints were accompanied by many histological alterations. The experiment revealed the toxic visceral effects of Leflunomide on the liver, heart and kidney.
\end{abstract}

Keywords

ARAVA $^{\circledR}$ (Leflunomide), Rheumatoid Arthritis, Side Effects

\section{Introduction}

Due to their incidence and severity, chronic inflammatory rheumatisms are one of the major public health problems [1]. These conditions affect the whole body, as they impede on the quality of life of the patients and often

"Corresponding author.

How to cite this paper: Manole, M., Badescu, C., Ciocoiu, M., Badescu, M. and Chicu, M. (2014) Arava Treatment, between Beneficial Action on Joint Inflammation and Side Effects on Liver, Myocardium and Kidney in Experimental Murine Arthritis. J. Biomedical Science and Engineering, 7, 712-720. http://dx.doi.org/10.4236/jbise.2014.79070 
lead to death [2]. Despite the numerous attempts of identification of rheumatoid arthritis etiology, it is still not fully known [3]. The cytokine family, and especially $\mathrm{IL}_{1 \alpha}, \mathrm{IL}_{1 \beta}$ and TNF- $\alpha$ [4] [5] are thought to play an important part in rheumatoid arthritis pathogenesis [6]. Rheumatoid arthritis therapy has undergone radical changes these last few years, its main goal being disease amelioration, structural joint deterioration reduction and joint functioning improvement [7]. There are drugs that are able to stop the disease from progressing, that may ensure good joint functioning and improve the patient's quality of life. Therefore, about $40 \%$ of the early diagnosed cases of rheumatoid arthritis may be cured.

Leflunomide (Arava) is the only orally administered drug for rheumatoid arthritis that seems to be associated with the best long lasting responses [8]. Leflunomide is a drug used to treat swelling and pain in arthritis [9]. It belongs to a class of medications called disease-modifying antirheumatic drugs (DMARDs). This class of medicines can decrease joint damage and disability. Leflunomide reduces inflammation in the joints that is responsible for both the symptoms of rheumatoid arthritis and the destruction of joints [10]. Leflunomide reduces inflammation by suppressing the activity of immune cells responsible for the inflammation [11]. Leflunomide is an immunomodulatory drug that achieves its effects by inhibiting the mitochondrial enzyme dihydroorotate dehydrogenase (DHODH) — an enzyme involved in de novo pyrimidine synthesis—which plays a key role in the de novo synthesis of the uridine monophosphate (rUMP), which is required for the synthesis of DNA and RNA [12]. Without DNA and RNA the immune cells (and most other types of cells) cannot multiply or function (or exist). The inhibition of human DHODH by teriflunomide, the active metabolite of leflunomide, occurs at levels (approximately $600 \mathrm{nM}$ ) that are achieved during treatment of rheumatoid arthritis (RA). Teriflunomide also inhibits several tyrosine kinases [12]. Because of its unique and different mechanism of action, Leflunomide is of value when added to other medications used for treating rheumatoid arthritis.

\section{Materials and Methods}

The experimental study was conducted on female Wistar rats. We preferred female rats since rheumatoid arthritis is considerably more common in females. Chronic arthritis was induced by $1 \%$ carrageenan solution injected in the knee joint for 8 weeks [13]. The occurrence of inflammatory arthritis and the effects of Leflunomide on the evolution of the joint inflammation phenomena were followed by successive radiological, biochemical and osteodensimetric determinations [14]. The reason for which we preferred this drug was that so far it is the only medication with good clinical long-term results, but also with a series of adverse effects.

The knee joint and various organs, such as liver, kidney and heart, were sampled at the end of the experiment in order to reveal any possible side effects of Leflunomide and carrageenan, respectively. All the experimental procedures conducted on the animals included in this study strictly complied with the international ethical regulations. At the end of the study, the animals were euthanized under ether anesthesia. Their death was quick and painless. When the animals stopped showing any vital signs (breathing, heart beats, reflexes), they were dissected and we sampled their knee joints, liver, heart and kidney (Andrews, E. J., AVMA Euthanasia Protocol, 1993).

Experimental Design. Animals were randomized into three groups:

Group $\mathbf{M}-(\mathrm{n}=12)$ the control group, which included twelve 4.5 months (18 weeks) old animals weighing 300-350g;

Group A- $(\mathrm{n}=12)$ the group of animals to which arthritis was induced by $0.02 \mathrm{ml}$ of $1 \%$ carrageen an solution injected in their knee joint 3 times/week, for 8 weeks;

Group $\mathbf{T}-(\mathrm{n}=12)$ the group of animals to which arthritis was induced by $0.02 \mathrm{ml}$ of $1 \%$ carrageen an solution injected in their knee joint 3 times/week, for 8 weeks and treated with $20 \mathrm{mg} / \mathrm{kg}$ of body weight of Leflunomide (Arava) force fed once every 2 days, for 8 weeks.

The animals were kept in plastic cages (a maximum of 4 rats per cage), in a room with controlled temperature $\left(24^{\circ} \mathrm{C}-28^{\circ} \mathrm{C}\right.$ ) and luminosity (a $12 \mathrm{~h}$ light: $12 \mathrm{~h}$ dark cycle) with free access to water and balanced foodrations. The animals were kept in accordance to the guidelines of the Committee on Care and Use of Laboratory Animals of the National Research Council of the National Institutes of Health.

\section{Results and Discussion}

Correlation intensity, both towards adult control rats and between the two study moments, reveals the mean severity of the forms of arthritis in the group of adult animals treated with Leflunomide, which supports the effec- 
tiveness of the therapy (Figure 1).

The hematological parameters showed strong statistically significant variations between the group of animals under Leflunomide therapy and the group of animals with chronic arthritis not receiving any treatment as well as the control group. The hemoglobin, erythrocyte and platelet levels dropped significantly in arthritic animals. The leukocyte level increased.

The $\mathrm{IL}_{1 \beta}$, fibrinogen, ceruloplasmin and uric acid levels were significantly higher in untreated animals than in animals under Leflunomide therapy, which reveals the protective effect of Leflunomide on the inflammatory carrageenan-induced bone and joint destruction.

\section{Post Mortem Examination}

Rat joint, liver, kidney and myocardium were sampled and the specimens were fixed in $10 \%$ formalin solution and paraffin embedded using a routine technique. The joints previously underwent a decalcification process in $15 \%$ trichloro-acetic acid solution. The specimens obtained were hematoxylin and eosin stained (HE) and prepared using the van Gieson method. When HE staining revealed myocardium muscle fiber injuries, we resorted to Lie staining.

The appearance of the knee joint is normal in the animals included in the control group: free joint space, intact joint cartilage surface, normal sized non-mineralized and mineralized cartilage.

Signs of erosive chronic arthritis were found in all the animals included in the group of animals in which experimental arthritis was induced [15]. The two cartilage areas (non-mineralized and mineralized) appeared thinner, frequently disorganized, eroded and replaced by the inflammatory pannus. Reduced subchondral bony plate, and abundant inflammatory pannus in the joint cavity (Figure 2, Figure 3). We also found areas of bone resorption in the subchondral bony plate (Figure 4, Figure 5). The amount of intra-articular pannus varied, either partially invading the joint cavity, or fully obliterating the joint cavity. The hypertrophied synovial was thicker and hypertrophied, and the underlying adipose tissue was invaded by inflammatory cells [16].

The arthritic lesions of all the arthritic animals under Arava therapywere smaller, yet the intra-articular pannus still had extended areas of fibrinoid necrosis, which even turned to small abscesses and leukocytic infiltrates. Limited bone resorption is associated with early fibrosis lesions (Figure 6, Figure 7).

Micro- and macro-vesicular lipid dystrophy lesions, numerous apoptosis cells and inflammatory infiltrates were detected in the interstitial and/or portal spaces in the liver of most animals receiving Arava therapy (Figure 8, Figure 9).

Important lesions were detected in the heart, namely interstitial and perivascular inflammatory chronic infiltrates associated with interstitial fibrosis lesions (Figure 10, Figure 11). Here and there we found traces of myocardium fiber tumefaction, disappearing streaks, accompanied by nodular inflammatory infiltrate (Figure 12); many of the swollen fibers were Lie stain positive, which reveals an early necrosis process (Figure 13).

Kidney lesions were isolated and were represented by discrete chronic interstitial inflammatory infiltrates (Figure 14).

The signaled importance of liver and cardiac lesions, which support the toxicity of the agent under survey (Leflunomide): lipid dystrophy, apoptosis cells and chronic inflammatory hepatic infiltrates and chronic inflammatory interstitial infiltrates, with isolated muscle cells affected by necrosis, as well as interstitial fibrosis in the heart. Leflunomide was approved by the FDA in September 1998. Leflunomide was approved for use in rheumatoid arthritis and has been used in over 200,000 patients since [17]. The safety profile was established by several studies, where in the most common reported adverse events included diarrhea, dyspepsia, rash, hair loss, elevated hepatic enzymes and hypertension [18].

\section{Conclusions}

Our animal trial (on female Wistar rats) consisted of an experimental model of inflammatory chronic arthritis induced by $1 \%$ carrageenan solution injection in the knee joint. The clinical appearance of non-specific inflamematory chronic arthritis was revealed by radiological, biochemical, immunological, hematological and postmortem exams. The morphological lesions occurring in adult arthritic animals were suggestive of erosive chronic arthritis, with cartilaginous area disorganization, replacement by and covering with inflammatory pannus. The joint space was filled with inflammatory pannus, and the underlying trabecular bone showed signs of osteolysis lesions. 


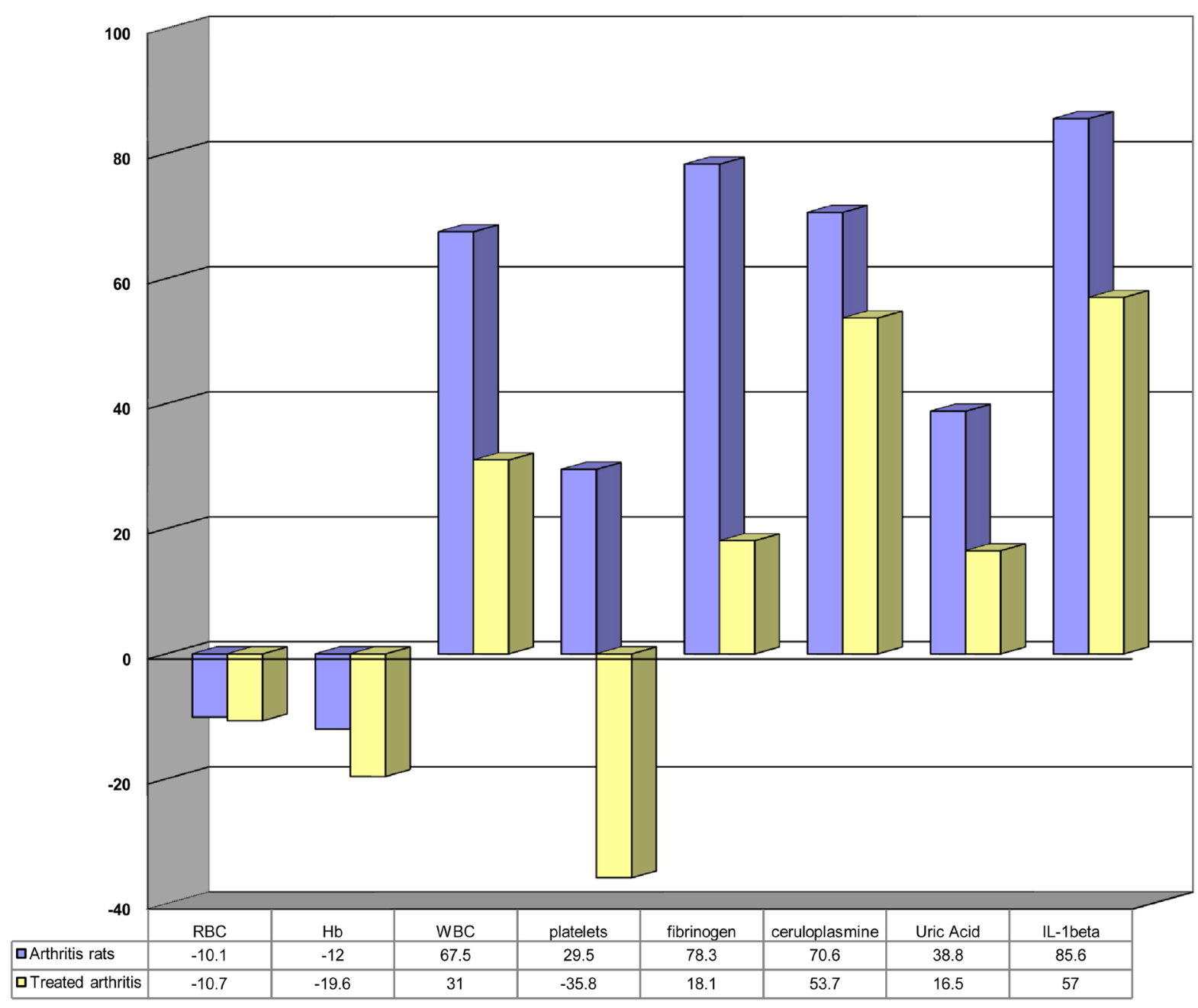

Figure 1. Analyzed parameters in the group of arthritic animals under Leflunomide therapy and in the group of arthritic animals not getting any treatment (compared to control animals).

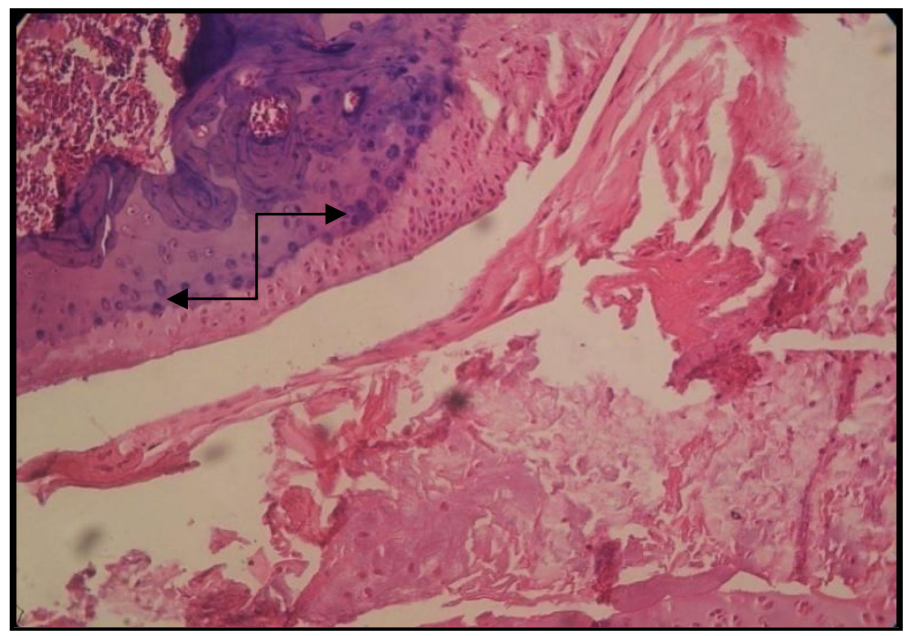

Figure 2. Arthritic rat. Joint cross section. Col. HE. ob. 10. Disorganized non-mineralized cartilage. Reduced subchondral bony plate (black arrow) with resorption areas, abundant intra-articular pannus. 


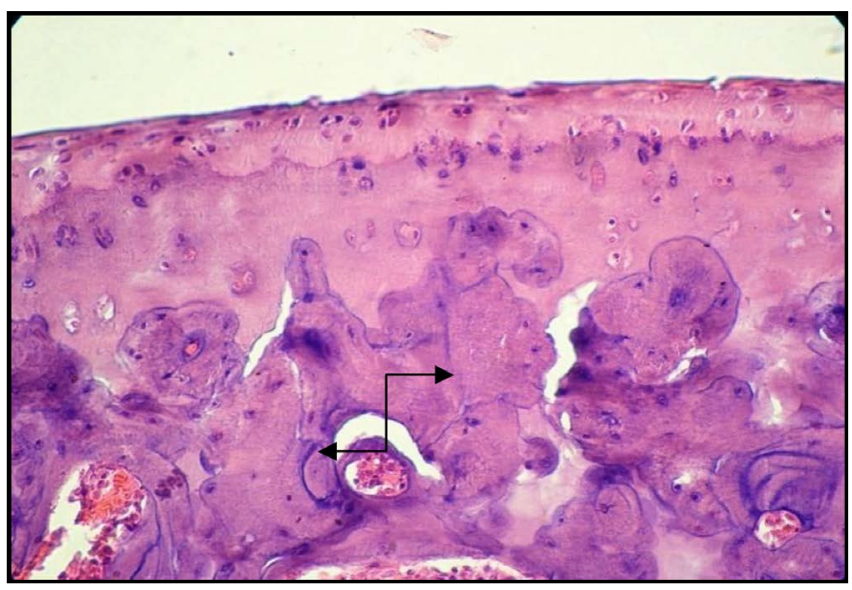

Figure 3. Arthritic rat. Joint cross section. Col. HE. ob. 40. Thickened and disorganized cartilaginous areas. Reduced subchondral bony plate, with resorption areas (black arrow).

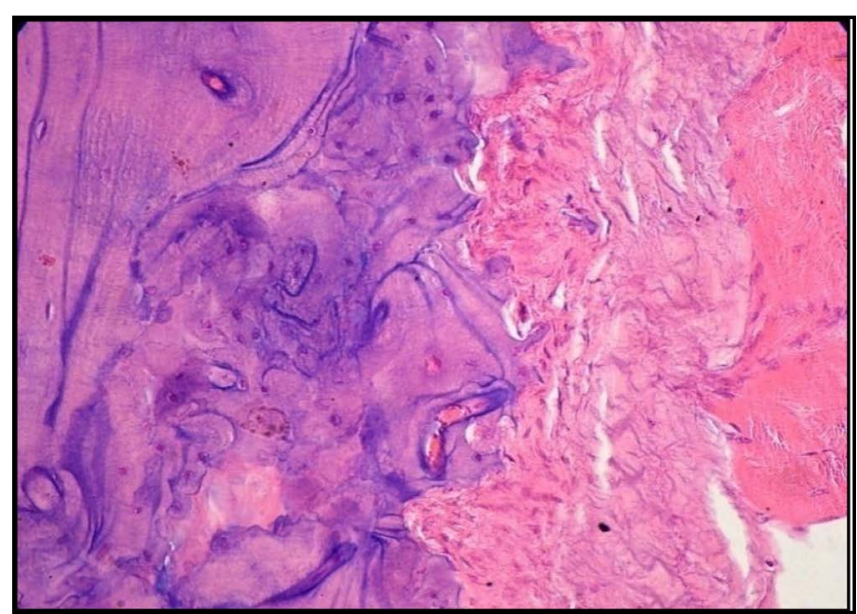

Figure 4. Arthritic rat. Joint cross section. Col. HE. ob. 40. Bone eroding by pannus.

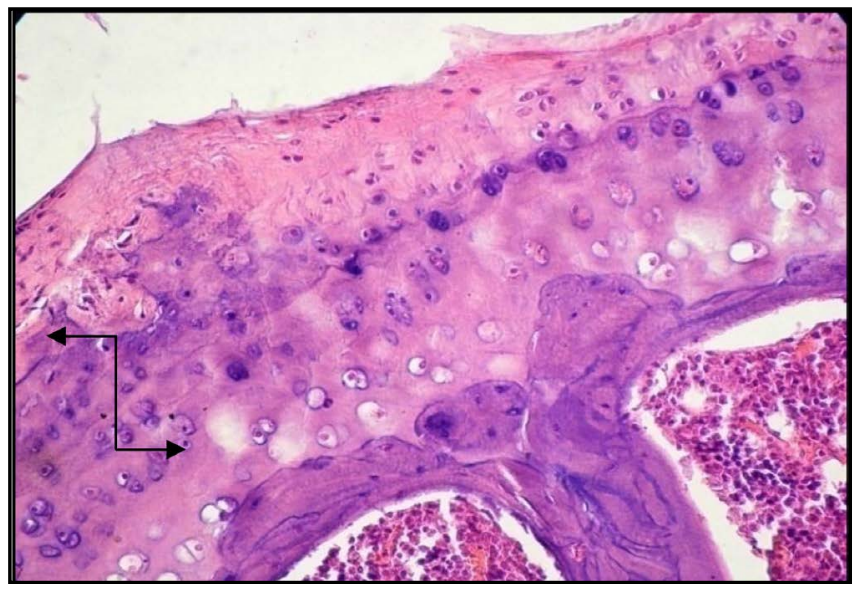

Figure 5. Arthritic rat. Joint cross section. Col. HE. ob. 40. Important cartilaginous area disorganization. Reduced subchondral bony plate, with resorbtion areas (black arrow). 


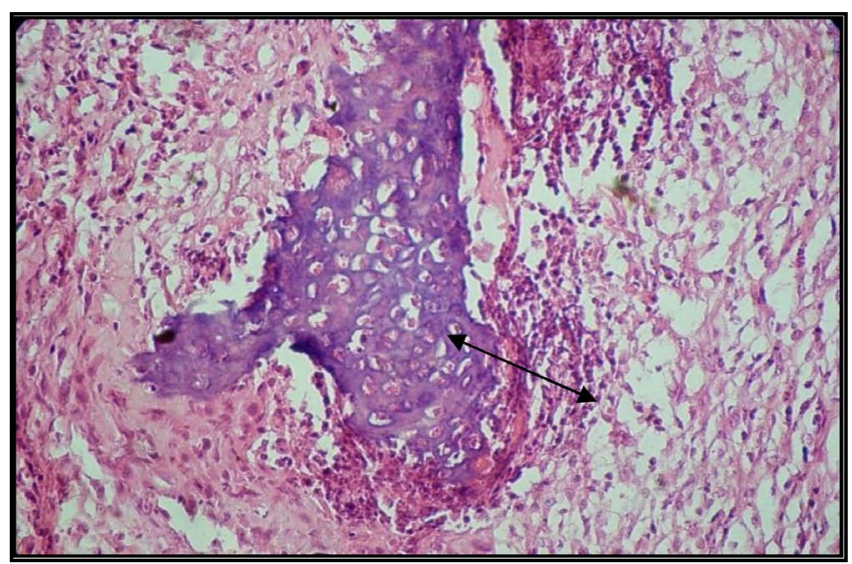

Figure 6. Arthritic rat receiving treatment. Joint cross section. Col. HE. ob. 40. Osteolysis area surrounded by inflammatory infiltrate and fibrosis process in progress (black arrow).

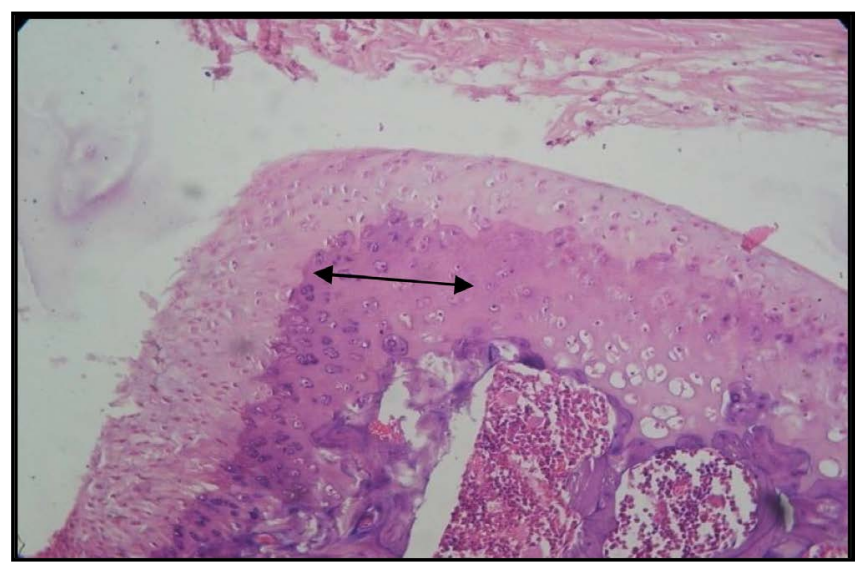

Figure 7. Arthritic rat receiving treatment. Joint cross section. Col. HE. ob. 20. Arthritic lesions with little intra-articular pannus and slight reduction of the cartilaginous areas (black arrow). Diminished subchondral bony plate.

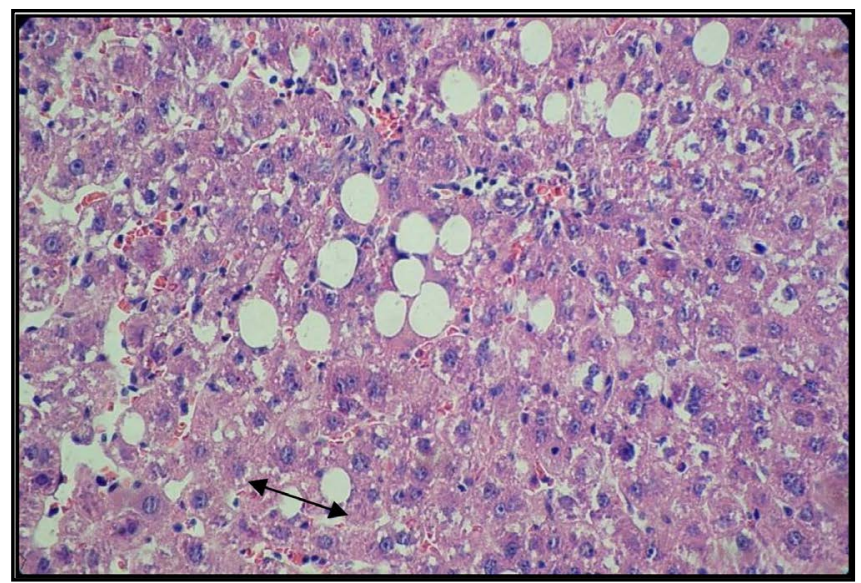

Figure 8. Liver of arthritic rat receiving Arava treatment. Col. HE. ob. 40. Fragmented micro and macro vesicular (black arrow) lipid dystrophy. 


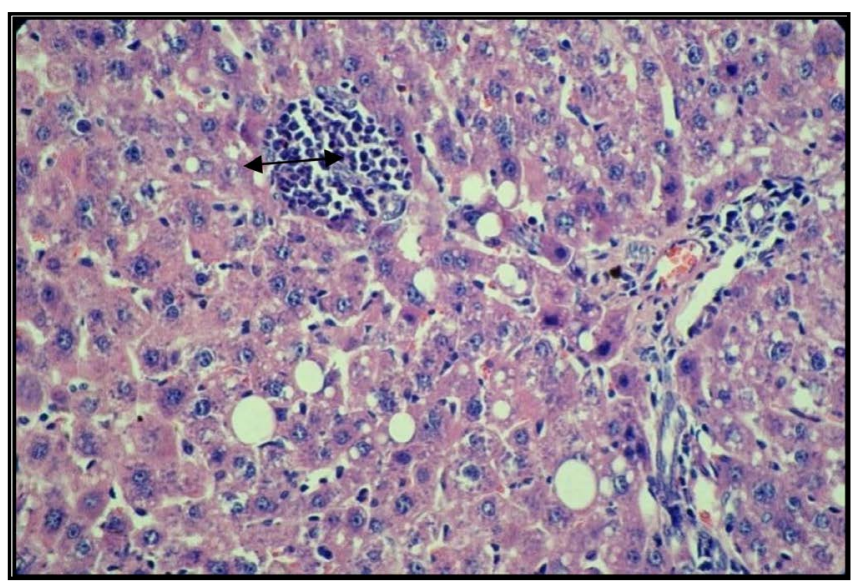

Figure 9. Liver of arthritic rat receiving Arava treatment. Col. HE. ob. 40. Lipid dystrophy, apoptosis cells, portal space and interstitial inflammatory infiltrate (black arrow).

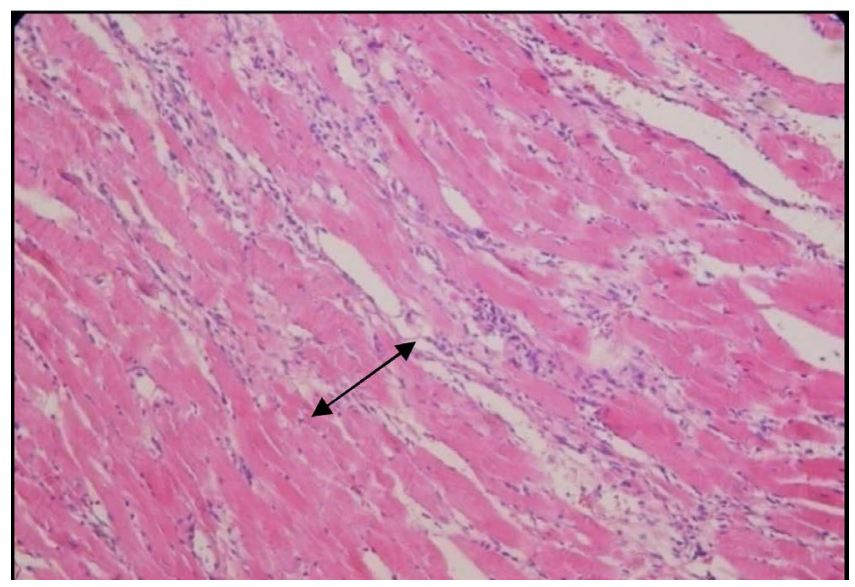

Figure 10. Heart of arthritic rat receiving Arava treatment. Col. HE. ob. 20. Interstitial inflammatory infiltrate among the myocardium fibers (black arrow).

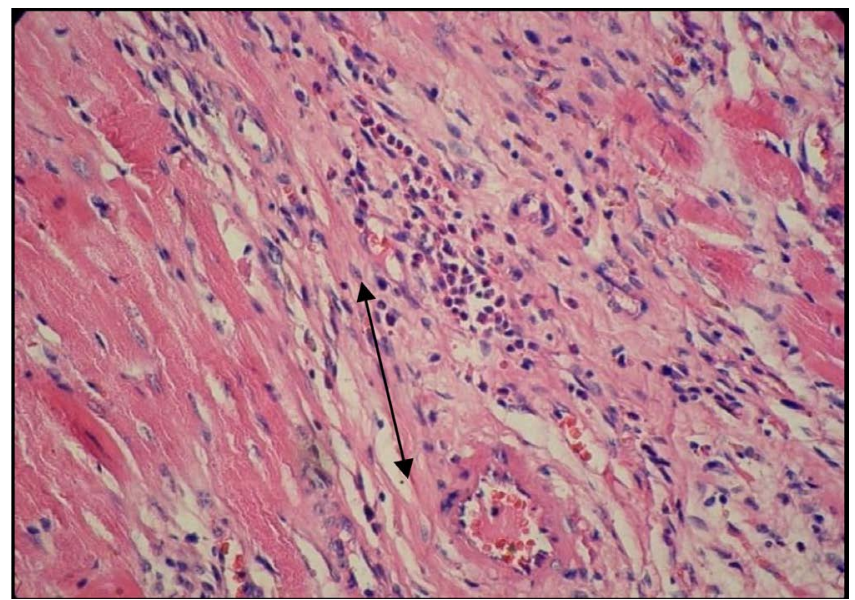

Figure 11. Heart of arthritic rat receiving Arava treatment. Col. HE. ob. 40. Interstitial and perivascular inflammatory infiltrate (black arrow). Early fibrosis. 


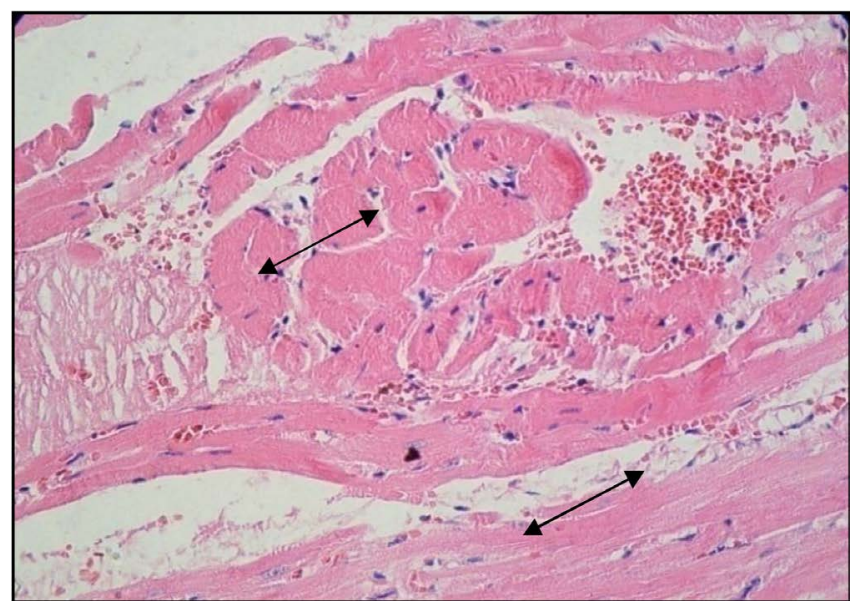

Figure 12. Heart of arthritic rat receiving Arava treatment. Col. HE. ob. 40. Myocardium fiber tumefaction and homogenization. Interstitial nodular inflammatory infiltrate (black arrow).

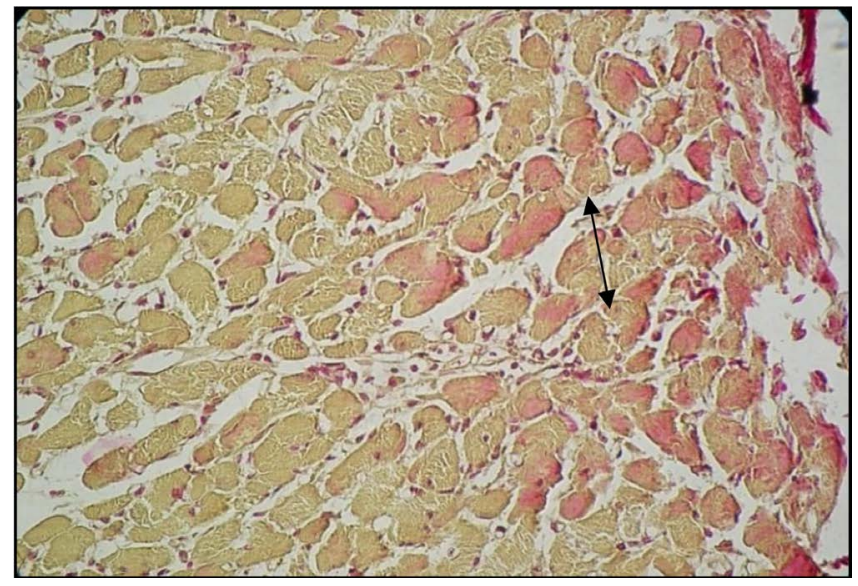

Figure 13. Heart of arthritic rat receiving Arava treatment. Lie stain. ob. 40. Necrotic myocardium fibers, Lie+ (black arrow).

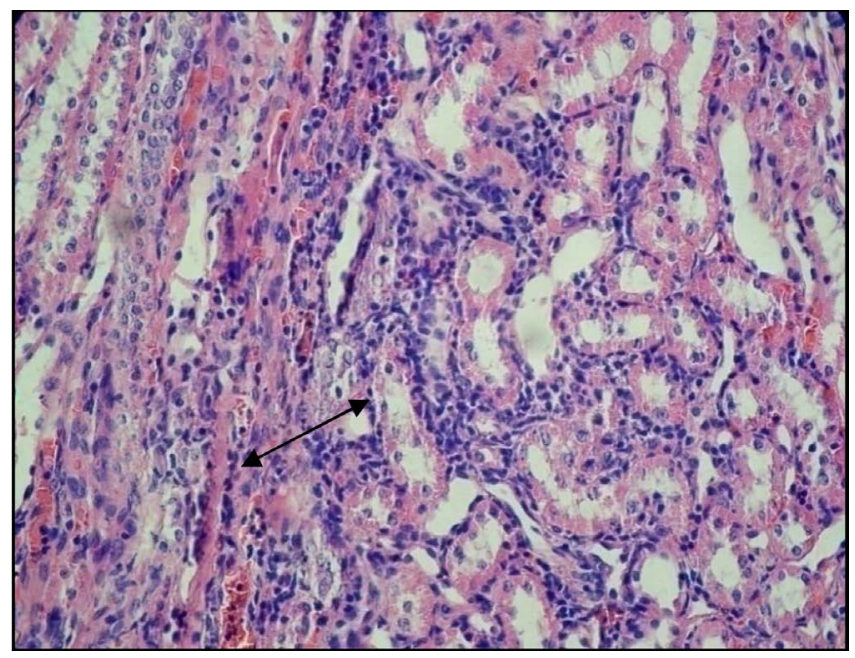

Figure 14. Kidney of arthritic rat receiving Arava treatment. Col. HE. ob. 40. Interstitial inflammatory infiltrate (black arrow). 
The response to Leflunomide therapy was undeniable. The phenomenon was revealed by radiological, biochemical and post-mortem exams. Leflunomide therapy improved the course of the clinical and paraclinical parameters, but it did not cure the condition. The weight loss suffered by the groups of animals under Leflunomide therapy confirmed the literature data on its anorexigenic effect.

The experiment revealed the toxic visceral effects of Leflunomideon the liver, heart and kidney.

- The liver was extremely affected in the groups of animals under Leflunomide therapy, which supports the literature data on its hepatic toxicity;

- We revealed the toxic effect of Leflunomide on the heart;

- The post-mortem kidney examination revealed minor changes.

The deaths occurred in the group of animals under Leflunomide therapy were due to various phenomena such as acute liver failure, hemorrhage, local infections, appetite diminution and consequent weight loss.

\section{References}

[1] Scott, D.L. (2000) Prognostic Factors in Early Rheumatoid Arhritis. Rheumatology, 39, 24-29. http://dx.doi.org/10.1093/oxfordjournals.rheumatology.a031490

[2] Firenstein, G. (1997) Ethiology and Pathogenesis of Rheumatoid Arthritis. Textbook of Rheumatology, Kelly \& Harris, 9, 1035-1086.

[3] Harris, E.D. (1999) Rheumatoid Arthritis: Pathophysiology and Implications for Therapy. New England Journal of Medicine, 322, 1277-1289.

[4] Burmester, G.R., Stuhlmuller, B., Keyszer, G. and Kinne, R.W. (1999) Mononuclear Phagocytes and Rheumatoid Arthritis. Mastermind or Workhorse in Arthritis? Arhritis Rheumatology, 40, 5-18. http://dx.doi.org/10.1002/art.1780400104

[5] Feldman, M., Brennan, F.M. and Maini, R.N. (2006) Role of Cytokines in Rheumatoid Arthritis. Annual Review of Immunolology, 14, 397-440. http://dx.doi.org/10.1146/annurev.immunol.14.1.397

[6] Bresnihan, B. (1999) Pathogenesis of Joint Damage in Rheumatoid Arthritis. Journal of Rheumatology, 26, 717-719.

[7] Coste, J., Spira, A., Clerc, D. and Paolaggi, J.B. (2007) Prediction of Articular Destruction in Rheumatoid Arthritis: Disease Activity Markers Revisited. Journal of Rheumatology, 24, 28-34.

[8] Lee, S., Park, Y., Park, J., Kang, Y., Nam, E., Kim, S., Lee, J., Yoo, W. and Lee, S. (2009) Combination Treatment with Leflunomide and Methotrexate for Patients with Active Rheumatoid Arthritis. Scandinavian Journal of Rheumatology, 38, 11-14. http://dx.doi.org/10.1080/03009740802360632

[9] Pinto, P. and Dougados, M. (2006) Leflunomide in Clinical Practice. Acta Reumatológica Portuguesa, 31, $215-224$.

[10] Sanders, S. and Harisdangkul, V. (2002) Leflunomide for the Treatment of Rheumatoid Arthritis and Autoimmunity. American Journal of Medical Sciences, 323, 190-193. http://dx.doi.org/10.1097/00000441-200204000-00004

[11] Scott, D.L. and Strand, V. (1997) Leflunomide a New Immunosupressive Drug. Arthritis \& Rheumatology, 67, 23512359.

[12] Teschner, S. and Burst, V. (2010) Leflunomide: A Drug with a Potential beyond Rheumatology. Immunotherapy, 2, 637-650. http://dx.doi.org/10.2217/imt.10.52

[13] Harrison, B. and Symmons, A. (2000) Early Inflammatory Polyarthrtis: Results from the Norfolk Arthritis Register with a Review of the Literature. Rheumatology, 39, 939-949. http://dx.doi.org/10.1093/rheumatology/39.9.939

[14] Zijistra, T.R., Bernelot Moens H.J. and Bukhari, M.A.S. (2001) The Rheumatoid Arthritis Articular Damage Scor. Annals of the Rheumatic Diseases, 60, 20-23.

[15] Firenstein, G. (1996) Invasive Fibroblast-Like Synoviocytes in Rheumatoid Arthritis. Passive Responders or Transformed Aggressors. Arthritis Rheumology, 39, 1781-1790. http://dx.doi.org/10.1002/art.1780391103

[16] Yanni, G., Whelan, A., Feighery, C. and Bresnihan, B. (1994) Synovial Tissue Macrophages and Joint Erosion in Rheumatoid Arthritis. Annals of the Rheumatic Diseases, 53, 39-44. http://dx.doi.org/10.1136/ard.53.1.39

[17] Unizony, S., Stone, J.H. and Stone, J.R. (2013) New Treatment Strategies in Large-Vessel Vasculitis. Current Opinion in Rheumatology, 25, 3-9. http://dx.doi.org/10.1097/BOR.0b013e32835b133a

[18] Steinbrocker, O., Trager, G.H. and Butterman, R.C. (1994) Therapeutic Criteria in Rheumatoid Arthritis. JAMA, 140, 659-662. http://dx.doi.org/10.1001/jama.1949.02900430001001 
Scientific Research Publishing (SCIRP) is one of the largest Open Access journal publishers. It is currently publishing more than 200 open access, online, peer-reviewed journals covering a wide range of academic disciplines. SCIRP serves the worldwide academic communities and contributes to the progress and application of science with its publication.

Other selected journals from SCIRP are listed as below. Submit your manuscript to us via either submit@scirp.org or Online Submission Portal.
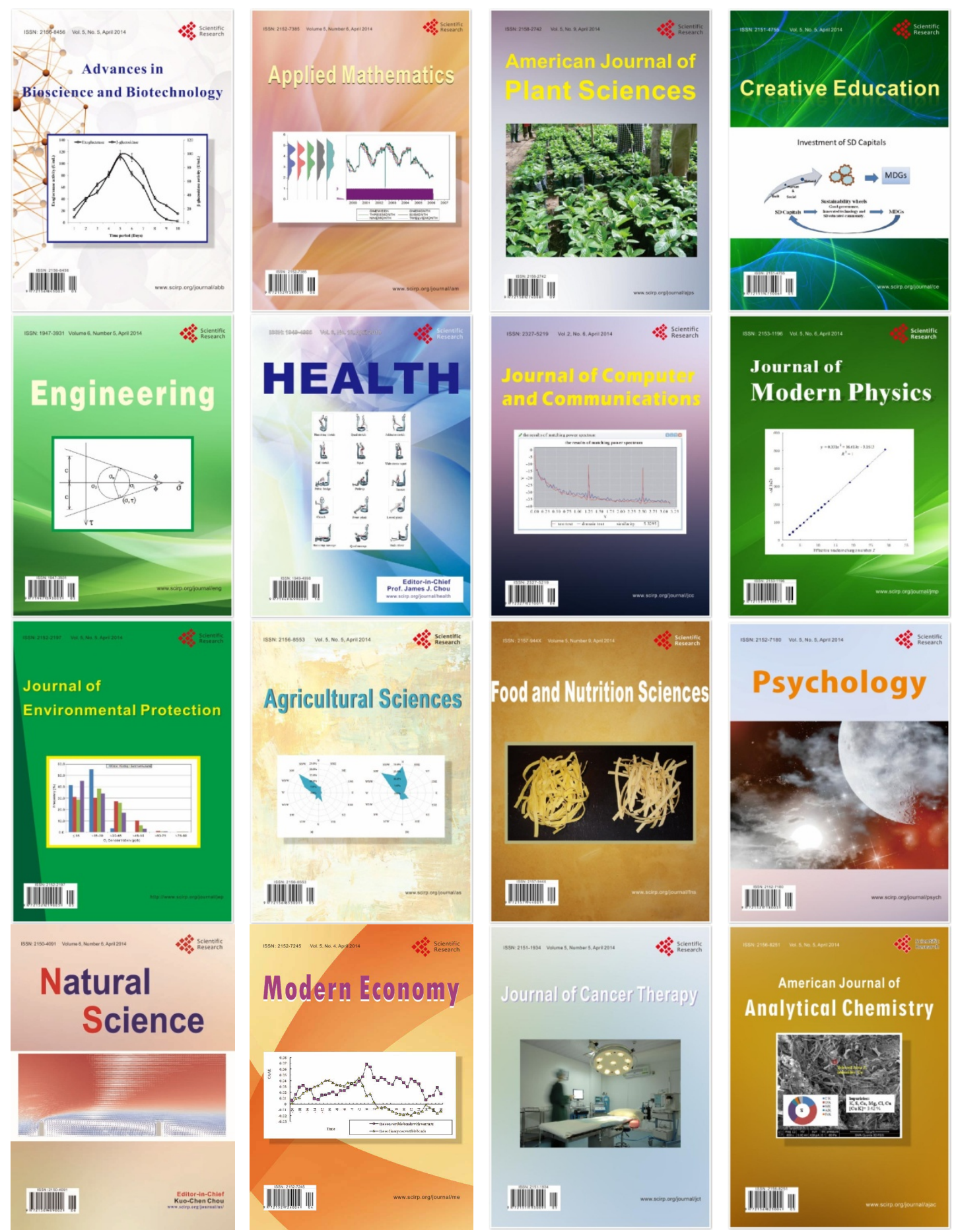